\title{
ELETROCONVULSOTERAPIA EM IDOSOS DEPRIMIDOS: RELATO DE EXPERIÊNCIA
}

Zeyne Alves Pires Scherer ${ }^{1}$, Edson Arthur Scherer ${ }^{2}$

\begin{abstract}
RESUMO: Neste estudo apresentamos aspectos referentes à eletroconvulsoterapia como recurso terapêutico em idosos que sofrem de depressão grave, com base no relato de nossa experiência. Objetivamos descrever a participação da equipe no procedimento da eletroconvulsoterapia e os recursos utilizados no ensino de estudantes de graduação e pós-graudação no cenário de prática. O grau de limitação associado à depressão geriátrica requer intervenção rápida, que é possibilitada pela eletroconvulsoterapia, procedimento eficaz e seguro. Na nossa prática, percebemos uma mudança nos aprendizes em relação aos conhecimentos, habilidades e atitudes adquiridos, quando "mitos são quebrados" e um novo conhecimento é construído. A eletroconvulsoterapia passa a ser reconhecida como uma estratégia terapêutica válida, útil e possível em Psiquiatria. Portanto, sugerimos a implementação de programas de ensino e de atualização permanente sobre a temática apresentada, tanto para estudantes quanto para profissionais da área da saúde.
\end{abstract}

PALAVRAS-CHAVE: Eletroconvulsoterapia; Idoso; Depressão; Enfermagem.

\section{ELECTROCONVULSIVE THERAPY IN ELDERLY PERSONS WITH DEPRESSION: AN EXPERIENCE}

\begin{abstract}
In this study we present aspects relevant to electroconvulsive therapy as a therapeutic resource for elderly people suffering from deep depression, based in our experience. We aim to describe the participation of the team in the procedure of electroconvulsive therapy and the resources utilized in the teaching of undergraduate and post-graduate students in the practice setting. The degree of limitation associated with geriatric depression requires rapid intervention, which is made possible by electroconvulsive therapy, an efficient and safe procedure. In our practice, we notice a change in the trainees in relation to knowledge, abilities and attitudes acquired, as when 'myths are broken', new knowledge is constructed. Electroconvulsive therapy is becoming recognised as a therapeutic strategy which is valid, useful and possible in Psychiatry. Therefore, we suggest the implementation of teaching programs and continuous professional development on the subject presented, as much for students as for health care professionals.
\end{abstract}

KEYWORDS: Electroconvulsive therapy; Elderly; Depression; Nursing.

\section{ELETROCONVULSOTERAPIA EN ANCIANOS DEPRIMIDOS: INFORME DE EXPERIENCIA}

RESUMEN: En este estudio son presentados aspectos acerca de la eletroconvulsoterapia como recurso terapéutico en ancianos que sufren de depresión grave, con base en el informe de nuestra experiencia. Nuestro objetivo es describir la participación del equipo en el procedimiento de la eletroconvulsoterapia y los recursos utilizados en la enseñanza de estudiantes de graduación y posgrado en la práctica. El grado de limitación asociado a la depresión geriátrica necesita intervención rápida, la cual es posible por medio de la eletroconvulsoterapia, procedimiento eficaz y seguro. En nuestra práctica, percibimos un cambio en los aprendices acerca de los conicimientos, habilidades y actitudes adquiridos, cuando "mitos son rotos" y un nuevo conocimiento es construido. La eletroconvulsoterapia pasa a ser reconocida como una estrategia terapéutica válida, útil y posible en Psiquiatría. Por lo tanto, sugerimos la implementación de programas de enseñanza y de actualización permanente sobre la temática presentada, tanto para estudiantes cuanto para profesionales del área de la salud.

PALABRAS CLAVE: Eletroconvulsoterapia; Anciano; Depresión; Enfermería.

${ }^{1}$ Enfermeira. Doutora em Enfermagem Psiquiátrica. Professora do Departamento de Enfermagem Psiquiátrica e Ciências Humanas e do Programa de Pós-Graduação da Escola de Enfermagem de Ribeirão Preto da Universidade de São Paulo. ${ }^{2}$ Médico Psiquiatra. Doutor em Patologia Experimental. Professor do Curso de Medicina do Centro Universitário Barão de Mauá. Médico Assistente do Hospital das Clínicas de Ribeirão Preto da Universidade de São Paulo.

Autor correspondente:

Zeyne Alves Pires Scherer

Universidade de São Paulo

Av. Bandeirantes, 3900 - 14040-902 - Ribeirão Preto-SP-Brasil

Recebido: 03/11/10

E-mail: scherer@eerp.usp.br

Aprovado: 10/03/11 


\section{INTRODUÇÃO}

A depressão na população idosa pode ser considerada como um grave problema de saúde pública; tem alta prevalência, ocorre com outras doenças (câncer, cardiopatias, neuropatias, distúrbios metabólicos, artrite e perdas sensoriais), tem impacto negativo na qualidade de vida e carrega um alto risco de suicídio. O reconhecimento da depressão nesta população pode, também, ser mais difícil, levando clínicos e pacientes a considerar possíveis sintomas depressivos como consequências normais do processo de envelhecimento, ou ainda, como incapacidades que se instalam em decorrência das baixas expectativas relacionadas ao período pós-aposentadoria. A queixa de humor depressivo é menos comum, enquanto os sintomas somáticos, como perda do apetite, irritabilidade, insônia, dores e perda da energia, são mais proeminentes. Como resultado, a depressão em idosos é subdiagnosticada ${ }^{(1-4)}$. Além disso, pessoas com mais de 60 anos tendem a ser mais resistentes aos tratamentos, em parte devido às características de persistência e recorrência, e por outra devido às interações com medicamentos utilizados para tratar suas doenças sistêmicas ${ }^{(2-3,5-7)}$.

A depressão em idosos está associada, também, a maior comprometimento cognitivo e a alterações cerebrais, como atrofia cerebral e lesões da substância branca. Do mesmo modo, os fatores etiológicos incluem, além dos fatores psicossociais e genéticos, doenças cerebrovasculares e seus fatores de risco (hipertensão, diabetes, aumento dos lipídios séricos e fumo) $)^{(2,4,6,8)}$.

Para o tratamento da depressão em idosos, portanto, devem ser observados os aspectos sociais, familiares, psicológicos e físicos da pessoa. As recomendações clínicas para o tratamento desta clientela provêm de extrapolações de experiências com adultos jovens ou de meia idade, ou são baseadas em estudos com número pequeno de pessoas acima dos 60 anos. Há poucos estudos realizados com a população acima de 80 anos, um dos segmentos populacionais que mais rapidamente tem crescido. Além disto, estas pesquisas foram compostas por amostras pequenas ou não significativas. Portanto, conforme consenso na literatura especializada ${ }^{(2-4,9)}$, as duas categorias de tratamento para a depressão do idoso incluem a terapia biológica (farmacoterapia, eletroconvulsoterapia - ECT) e a terapia psicossocial (psicoterapia cognitiva, psicoterapia psicodinâmica e exercícios físicos). A maioria dos estudos disponíveis na literatura aborda o tratamento biológico.
Ao longo da nossa prática, contudo, temos percebido em contatos informais com colegas docentes e profissionais da área da saúde, inclusive da saúde mental, a falta de conhecimentos de alguns em relação à ECT e a presença de atitudes pré-conceituosas de oposição em relação à ECT. Da mesma forma, alunos (de graduação e pós-graduação na área da saúde) têm chegado ao cenário de prática com informações de caráter empírico em relação à ECT e, por vezes, manifestando opiniões de conteúdo preconceituoso e contrário à utilização desta modalidade terapêutica.

Diante destas observações julgamos oportuno apresentar aspectos referentes à ECT como recurso terapêutico em idosos que sofrem de depressão grave com base no relato de nossa experiência. Objetivamos descrever a participação da equipe de assistência de uma enfermaria psiquiátrica de hospital geral universitário no procedimento da ECT e os recursos utilizados no ensino de estudantes de graduação e pós-graduação na área da saúde no cenário de prática.

\section{ELETROCONVULSOTERAPIA NO IDOSO DEPRIMIDO}

O grau de limitação associado à depressão geriátrica requer, muitas vezes, uma intervenção rápida. A relativa ausência de interações medicamentosas, a resposta mais rápida à ECT comparada ao uso de psicofármacos, e os benefícios da intervenção precoce para a resposta terapêutica fazem a ECT, frequentemente, ser selecionada como primeira escolha no tratamento de depressão severa associada, ou não, à demência em pessoas idosas ${ }^{(10-11)}$.

Nos idosos acometidos por depressão grave, ou que sofrem de condições médicas comórbidas, ou que tenham baixa tolerância aos medicamentos psicotrópicos, ou apresentam sintomas psicóticos ou outros associados ao quadro depressivo (presença de agitação e agressividade, anorexia importante, risco de suicídio) e tenham história de boa resposta anterior à ECT, esta passa a ser o tratamento de escolha ${ }^{(10-13)}$.

A eficácia e a segurança do tratamento de idosos com a ECT são baseadas em estudos naturalísticos, retrospectivos (não randomizados) ou com número pequeno de sujeitos ${ }^{(8,14)}$. Um destes estudos ${ }^{(7)}$, realizado em Amsterdam (Holanda), mostrou evidências de que a terapia eletroconvulsiva pode aumentar o funcionamento cognitivo (memória, percepção, processamento de informações e funcionamento executivo) em idosos não demenciados. 
No Brasil, a Resolução do Conselho Federal de Medicina (Resolução CFM n. 1.640/2002), dispõe e dá outras providências sobre a ECT, reconhecendo ser um método terapêutico eficaz, seguro, internacionalmente reconhecido e aceito, que deve ser realizada em ambiente hospitalar. A resolução também considera que a ECT é um ato médico e que necessita do consentimento informado obtido do paciente, sua família ou responsáveis, por escrito, antes do início do tratamento. Dispõe, ainda, sobre indicações e cuidados necessários para este procedimento.

Os profissionais envolvidos com o procedimento devem observar possíveis alterações na condição clínica do idoso e que possam interferir na continuidade do tratamento eletroconvulsivo. Entre estas, encontram-se o agravamento de doenças cardiológicas, a ocorrência de acidentes vasculares cerebrais e o surgimento de lesões intracranianas expansivas, com aumento da pressão intracraniana ${ }^{(8,15)}$.

\section{ELETROCONVULSOTERAPIA NO IDOSO: RELATO DE EXPERIÊNCIA}

Em nossa experiência profissional enquanto educadores (de graduandos e pós-graduandos da área da saúde e profissionais da área da saúde mental), com frequência nos deparamos com pessoas idosas com quadros depressivos graves, resistentes aos tratamentos psicofarmacológicos tradicionais, bem como às intervenções psicossociais. Nestes casos, como a literatura recomenda, está indicado o tratamento com a $\mathrm{ECT}^{(2-}$ 3,7,10-13), o qual é realizado na unidade de internação psiquiátrica do hospital universitário onde exercemos nossas atividades.

A indicação para a ECT é discutida em reuniões clínicas, das quais participam os integrantes da equipe interdisciplinar de assistência (médico, enfermeiro, psicólogo, assistente social, terapeuta ocupacional e estagiários - graduandos e pós-graduandos de Medicina, Enfermagem, Psicologia, Terapia Ocupacional e Serviço Social). Uma vez indicada, é realizada reunião com o paciente e seus familiares para esclarecê-los sobre a indicação, implicações e os procedimentos a serem seguidos na realização da ECT. Ao concordarem, são convidados a assinar o Termo de Consentimento Livre e Esclarecido.

A ECT é realizada em sala equipada para tal, mediante equipe treinada (médicos psiquiatras - docente, assistente e residente; médico anestesiologista; enfermeiro e auxiliar de enfermagem). O procedimento é realizado sob anestesia e é conhecido como "ECT modificada", em contraste com os tratamentos sem anestesia ou relaxantes musculares do passado ${ }^{(13-18)}$. Por ser, portanto, um serviço formador e modelo para futuros profissionais, todas as medidas e cuidados sugeridos e descritos na literatura pertinente e na Resolução CFM n. 1.640/2002 em relação a este procedimento, nesta população, são seguidos.

Na nossa experiência junto aos estudantes (graduandos e pós-graduandos da área da saúde) adotamos como primeira conduta a de orientá-los a buscar na literatura esclarecimentos acerca da ECT. Em seguida, eles participam, junto com a equipe de assistência da unidade, dos procedimentos prévios a sua aplicação. $\mathrm{Na}$ sequência, observam-na e acompanham a equipe nos cuidados pós-procedimento. Cabe lembrar que todas estas atividades desenvolvidas pelos aprendizes são acompanhadas mediante discussão e avaliação do aprendizado.

O processo de ensino-aprendizagem adotado, ou seja, a combinação de elementos da problematização (dialógica) e da aprendizagem significativa (crítica reflexiva) tem propiciado manifestações positivas dos alunos. Eles mostram surpresa ao se deparar com as informações favoráveis à ECT que encontram em suas buscas na literatura; observam, também, o grau de conhecimento dos membros da equipe de assistência sobre esta modalidade de tratamento, fato que possibilita que cada um destes possa desempenhar seu papel profissional dentro dos preceitos do trabalho interdisciplinar. Os alunos manifestam surpresa quando o tratamento tem resposta eficaz e reconhecem ter sido válida a tentativa, mesmo quando o doente não responde favoravelmente.

Desta forma, é possível perceber uma mudança nos aprendizes em relação aos conhecimentos, habilidades e atitudes adquiridos. Quando mitos são quebrados e um novo conhecimento é construído, observamos que a ECT passa a ser reconhecida como uma estratégia terapêutica válida, útil e possível em Psiquiatria. Isto ocorre mesmo com futuros profissionais que optem por não trabalhar diretamente nesta área da saúde.

\section{CONSIDERAÇÕES FINAIS}

A apreensão de novas atitudes adquiridas em discussões e reflexões coletivas, como descrito neste estudo, mediante a articulação teórico-prática proporcionada no contato direto com a ECT em idosos severamente deprimidos, forneceu bases para que os 
estudantes desenvolvessem sua autonomia através de uma relação dialógica. O fato de a ECT ser, até hoje, um recurso cercado de polêmicas e mitos, valorizou ainda mais o conhecimento construído.

A mudança de postura evidenciada nestes alunos, tão almejada na formação dos futuros profissionais, foi percebida não somente em relação ao crescimento pessoal. As atitudes propulsoras de críticas, de participação e de competências adquiridas, também os instrumentalizaram para se tornarem agentes de transformação social. Portanto, apesar de poucos aprendizes terem a oportunidade de vivenciar este contato próximo com a ECT, acreditamos que estes possam ser disseminadores de conhecimento nos diversos cenários de prática (serviços, unidades de ensino, ou comunidade). Acreditamos, também, que são necessários esforços no sentido de promover programas de ensino e de atualização permanentes na temática apresentada, tanto para estudantes quanto para profissionais da área da saúde.

\section{REFERÊNCIAS}

1. Alvarenga MRM, Oliveira MAC, Faccenda O, Cerchiari EAN, Amendola F. Sintomas depressivos em idosos assistidos pela estratégia saúde da família. Cogitare Enferm. 2010;15(2):217-24.

2. Lebowitz BD, Pearson JL, Schneider LS, Reynolds CF, Alexopoulos GS, Bruce ML, et al. Diagnosis and treatment of depression in late life: consensus statement update. JAMA. 1997;278(14):1186-90.

3. Small GW. Pharmacotherapy and other treatments for elderly patients with depression. J Clin Psychiatry. 2010;71(1):e03.

4. Blazer DG. Psiquiatria geriátrica. In: Hales RE, Yudofsky SC. Tratado de psiquiatria clínica. Porto Alegre: Artmed; 2006. p. 1430-44.

5. Almeida OP. Manejo farmacológico da depressão em idosos. In: Lafer B, Almeida OP, Fráguas R, Miguel EC. Depressão no ciclo da vida. Porto Alegre:Artmed; 2000. p. 246-53.

6. Tier CG, Lunardi VLL, Santos SSC. Cuidado ao idoso deprimido e institucionalizado à luz da complexidade. Rev Eletr Enferm. [Internet] 2008;10(2) [acesso em 14 ago 2009]. Disponível: http://www.fen.ufg.br/revista/ v10/n2/v10n2a24.htm

7. Bosboom PR, Deijen JB. Age-related cognitive effects of ECT and ECT-induced mood improvement in depressive patients. Depression and Anxiety 2006; 23:93-101.

8. Van der Wurff FB, Stek ML, Hoogendijk WJG, Beekman ATF. The efficacy and safety of ECT on depressed older adults, a literature review. Int J Geriatr Psychiatry. 2003;18:894-904.

9. Frazer CJ, Christensen H, Griffiths KM. Effectiveness of treatments for depression in older people. Med J Aust. 2005;182(12):627-32.

10. Tew JD, Mulsant BH, Haskett RF, Prudic J, Thase ME, Crowe RR, et al. Acute efficacy of ECT in the treatment of major depression in the old-old. Am J Psychiatry. 1999; 156:1865-70.

11. Stoppe AJr. Depressão em idosos: guia para diagnóstico e conduta. São Paulo: Lemos; 2004.

12. Birkenhäger TK, Pluijms EM, Ju MR, Mulder PG, Van den Broek WW. Influence of age on the efficacy of electroconvulsive therapy in major depression: a retrospective study. J Affect Disord. 2010;126:257-61.

13. American Psychiatric Association. The practice of electroconvulsive therapy: recommendations for treatment, training and privileging. A task force report of the American Psychiatric Association. Washington: American Psychiatric Association; 2001.

14. Moreno DH, Moreno RA. Eletroconvulsoterapia. In: Lafer B, Almeida OP, Fráguas R, Miguel EC. Depressão no ciclo da vida. Porto Alegre: Artmed; 2000. p. 213-25.

15. Stuart GW. Terapias somáticas. In: Stuart GW, Laraia MT, organizadores. Enfermagem psiquiátrica: princípios e prática. Porto Alegre: Artmed; 2001. p. 641-53.

16. McCall WV. Electroconvulsive therapy in the era of modern psychopharmacology. Int J Neuropsychopharmacol. 2001;4:315-24.

17. Marangell LB, Silver JM, Goff DC, Yudofsky SC. Psicofarmacologia e eletroconvulsoterapia. In: Hales RE, Yudofsky SC. Tratado de psiquiatria clínica. Porto Alegre: Artmed; 2006. p. 985-1078.

18. Fitzsimons LM, Mayer RL. Soaring beyond the cuckoo's nest: health care reform and ECT. J Psychosoc Nurs Ment Health Serv. 1995;33(12):10-3. 